\title{
THE USE OF THE REFRACTIONAL PAIR OBSERVATIONS
}

\author{
G. TELEKI \\ Astronomical Observatory, Belgrade, Yugoslavia
}

\begin{abstract}
The author propose the inclusion in observational program of latitude services a number of Talcott pairs with greater zenithal distances (refraction pairs). It is specially advisable for the stations, forming a chain, where the comparison of local latitude data is necessary. Refraction pairs could be very useful for investigations of the local anomalous refraction influences.
\end{abstract}

The first observational program of the ILS was compiled so that in every $2 \mathrm{hr}$ group of Talcott pairs of stars there were six pairs with mean zenithal distances up to $24^{\circ}$ (latitude pairs) and two pairs with mean zenithal distances of about $60^{\circ}$ (refractional pairs). These refractional pairs were eliminated from the program of the ILS at the beginning of 1906.

We used (Teleki, 1970a) the results of the observations of these refractional pairs for the purpose of investigating local influences on the values of latitude at the stations of the ILS. In continuation of this investigation, we analysed (Teleki, 1970b) the results obtained from 24 latitude pairs of the ILS program, for the days for which we possessed also observations of refractional pairs.

It can be seen that the refractional pairs as well as the latitude pairs, give information about local influences on the values of latitude, but the information from the refractional pairs is more marked, which is very important and useful for the investigation of non-polar variations.

From the comparison of data of refractional and latitude pairs we may conclude that the influence of local factors increases with zenithal distance, but the size of the increase is different at different stations. There is no doubt that among these local factors, the influences of anomalous refraction are the most important ones. Therefore, refractional pairs could be very useful for investigations of the local influences of anomalous refraction. At this point, we do not contemplate the separation of the value of the influences of anomalous refraction out of the latitude measurements on the basis of a theoretical mathematical model of the influences of anomalous refraction which is common for all stations. Actually, it is impossible, and it is obvious that a special meteorological research is here needed. From these meteorological investigations we must obtain a mathematical model of the influence of anomalous refraction at the given station. The refractional pairs will be very useful for the control of this model.

Therefore our proposal is to include in the observational program of latitude services a number of Talcott pairs with greater zenithal distances (bigger than $45^{\circ}$ ). It is specially advisable for the stations, forming a chain, where the comparison of local latitude data is necessary. For this reason, we propose that the new ILS program, from the beginning of 1979 , should include a number of refractional pairs too. 


\section{References}

Teleki, G.: 1970a, Proc. Int. Lat. Obs. Mizusawa 10, 46-56.

Teleki, G.: 1970b, Seminarium 'Badania ruchu wirowego Ziemi', Warszawa, pp. 60-64.

\section{DISCUSSION}

T. Okuda: Is there any suggestion for an atmospheric model $t$ s explain the anomalous systematic increase or decrease in non-polar terms - for example in the case of Mizusawa we have -0!01 0!05/yr.

G. Teleki: Without special meteorological investigations it is impossible to propose any solution.

$S$. Débarbat: In the abstract of your paper you wrote "From these meteorological investigations we must obtain a mathematical model of anomalous refraction influence at the given station". What do you mean by a 'mathematical model'. We wish to propose another shape for the variations of the refraction.

G. Teleki: At present, we usually suppose that the form of the equal density layers is plane. It is a rough approximation only. Therefore I am sure that we can develop a mathematical model of the anomalous refraction influences for the given station only on the basis of the real meteorological measurements.

H. Enslin: (Query to Dr Yumi) Would it be possible to include 'refraction pairs' in the IPMS observing programme, from 1979 on?

Answer by Dr Melchior (Dr Yumi not present): This would be of high scientific value; the problem is to get observers to carry out such a programme. 